\title{
Analytical investigations of thermo-geometrical parameters effects on heat transfer and performance of longitudinal radiating fin
}

\author{
Bayo Yemisi Ogunmola, Gbeminiyi Sobamowo \\ Department of Mechanical Engineering, Faculty of Engineering, University of Lagos, Akoka, Lagos, Nigeria
}

Email address:

bayemi@yahoo.com (B. Y. Ogunmola),mikegbeminiyiprof@yahoo.com (G. Sobamowo)

\section{To cite this article:}

Bayo Yemisi Ogunmola, Gbeminiyi Sobamowo. Analytical Investigations of Thermo-Geometrical Parameters Effects on Heat Transfer and Performance of Longitudinal Radiating Fin. International Journal of Energy and Power Engineering.

Vol. 3, No. 3, 2014, pp. 139-146. doi: 10.11648/j.ijepe.20140303.15

\begin{abstract}
In this work, analytical models are developed for determining temperature distribution, heat transfer and the efficiency of radiating fin. The developed models are used to investigate the effects of various thermo-geometric properties on the temperature distribution and consequently on the performance of the radiating fin. The effects of fin tip conditions on the thermal performance of the fin are also examined. From the results, it is shown that the variation in the thermogeometric parameters (fin tip Biot number, modified Biot number and efficiency) of the fin has pronounced effects on the temperature distribution and consequently on the performance of the fin especially when a large temperature difference exists between the prime surface and the environment. The results obtained in this study serve as basis for determining the level of accuracy of any other approximation method use in the analysis of the problem and also, it could be used to improve the design of radiating fin in heat transfer equipment.
\end{abstract}

Keywords: Analytical Solution, Temperature Distribution, Heat Transfer, Thermo-geometric Parameters, Radiating Fin

\section{Introduction}

The ever growing demands for high-performance heat transfer components with progressively smaller weights, volume, costs or accommodating shapes have greatly increased the use of extended surfaces/fins to enhance heat dissipation loss from hot primary surfaces. The Power plants in the automobiles are becoming highly power-packed with increasing power to weight and/or volume ratio. The designs of radiator at present for the high-performance heat transfer components depend on the empirical methods, wherein existing experimental data are used as the rules of thumb for the design process. Also, the drive on automobile manufacturers for developing compact and energy efficient cars warrants a thorough optimization process in the design of all engine components, including radiators. Radiators are installed in automobiles to remove heat from the under hood which include engine cooling and heat removal during airconditioning process. The use of higher output engines with tightly packed under hood packaging demands a better understanding of the complex cooling fluid flow characteristics and resulting thermal performance of the radiator. Moreover, in the recent years, interest in fin with radiation heat transfer has been simulated in space exploration since radiation is almost the main mechanism by which waste heat from electronic part or other heat generating equipment in satellite can be dissipated. The enormous applications of this type of fin have aroused interest of various workers as there have been extensive works on the heat transfer characteristics, which attempt to improve the design and provide the optimized mass of the fins. In the attempts of studying the functions, investigating the heat transfer characteristics and optimizing the performance of the fin, Cockfield [1] discussed the role of radiators as a structural element in spacecraft applications while Wilkins Jr. [2] developed expressions for heat analysis of triangular fins to space at absolute zero temperature Few years later, Schnurr [3] optimized the design of longitudinal and triangular radiating fins with respect to weight. Meanwhile, workers such as Gopinath and Ashok [4] studied the problem of a uniform area radiating fin for large values of the radiation-conduction interaction parameter and developed for improving the previously known asymptotic solution to make it applicable for relatively smaller values of radiation conduction interaction parameter. Besides, Love and Francis [5] analyzed longitudinal radiating fins equally spaced 
around a cylindrical heat source, while Eslinger and Chung [6] presented a finite element solution for the heat transfer from a radiating and convecting fin or fin arrays. Schnurr et al [7] optimized the radiating fin arrays with respect to weight. Here, a non linear optimization approach was used to determine the minimum weight design for radiating fin arrays used in space applications and the analysis were brought out. In the following year, Truong [8] investigated the performance of radiating annular fins of various shapes while Karam and Eby [9] presented the linearised solution of conducting radiating fin. On the other hand, Chang [10] developed a computer model of a heat pipe radiator based on two correlations for the rectangular radiating fin efficiency. In the same vein, Zaulichnyi [11] analyzed the problem of heat transfer by means of radiators with finned heat pipes with onedimensional approximation and proposed an algorithm to design radiators of a specified capacity with constraints on their weight and dimension. Moreover, Sunil Kumar and Venkateshan [12] optimized tubular radiator with annular fins on a nonisothermal base. Few years later, Krikkis and Razelos [13] presented the correlations for optimum dimensions of longitudinal rectangular and triangular radiating fins with mutual irradiation. Neumann [14] has investigated analytically the thermal design of heat pipe/fin type space radiators for the case of uniformly tapered fins as well as for flat fins with constant thermal conductivity assumption. Equally, Cihat [15] evaluated the temperature distribution along a radiating fin by the analytical ADM (Adomian Decomposition Method) method while Hosseini et al [16] applied the HPM (Homotopy Perturbation Method) method for a variable thermal conductivity. In furtherance of this research, Bazdidi and Kamrava [17] provided a numerical calculation of temperature distribution analysis using finite volume technique for linear function of temperature-dependent thermal conductivity.

The heat transfer in radiating fin occurs nonlinearly and as such it is difficult to find the exact analytical solutions for such problems. Therefore, from the reviewed literatures, approximate analytical solutions were used among which homotopy perturbation method (HPM), Adomain decomposition method (ADM). The study (using either analytical or numerical methods) of thermo-geometrical parameter effects and the material thermal properties effects of a radiating fin and its heat transfer characteristics is limited in open literatures. Therefore, this work presents analytical solutions for the study of the effects of thermo-geometrical parameters, material thermal properties and the heat transfer characteristics on the performance of radiating fin.

\section{Model Assumptions and Development}

The following assumptions are made in the development of the governing equation

1. The heat flow in the fin and its temperatures remain constant with time. i.e. a steady state heat transfer.

2. The convective heat transfer coefficient on the faces of the fin is constant and uniform over the entire surface of the fin.
3. The temperature of the medium surrounding the fin is uniform.

4. The fin thickness is small, compared with its height and length, so that temperature gradients across the fin thickness and heat transfer from the edges of the fin may be neglected.

5. The fin base temperature is uniform.

6. There is no contact resistance where the base of the fin joins the prime surface.

7. There are no heat sources within the fin itself.

8. Heat transfer to or from the fin is proportional to the temperature excess between the fin and the surrounding medium.

Under these assumptions, the general differential equation of radiating fin is given by

$$
2 L f(x) \frac{d^{2} T}{d x^{2}}+\frac{2 L d f(x)}{d x} \frac{d T}{d x}-\frac{2 \sigma \varepsilon L\left(T^{4}-T_{\infty}^{4}\right)}{k}=0
$$

The profile function $f(x)$ for longitudinal fins usually will take the form

$$
f(x)=\frac{\delta_{o}}{2}\left(\frac{x}{b}\right)^{(1-2 n) /(1-n)}
$$

\subsection{Longitudinal Fin of Rectangular Profile}

For the longitudinal fin of rectangular profile displayed with its terminology and coordinate system in Fig. 2.1, the exponent on the general fin profile of Eq. (2) satisfies the geometry when $n=\frac{1}{2}$. The profile function for this fin then becomes

$$
f(x)=\frac{\delta_{o}}{2}
$$

Because $\delta \mathrm{b}=\delta$ and $\frac{d f(x)}{d x}=0$

On substituting Eq. (3) into Eq. (1), the governing differential equation becomes

$$
\frac{d^{2} T}{d x^{2}}-\frac{2 \sigma \varepsilon}{k \delta_{o}}\left(T^{4}-T_{\infty}^{4}\right)=0
$$

Which is an ordinary nonlinear second-order differential equation with constant coefficients.

\subsection{The Physical Model}

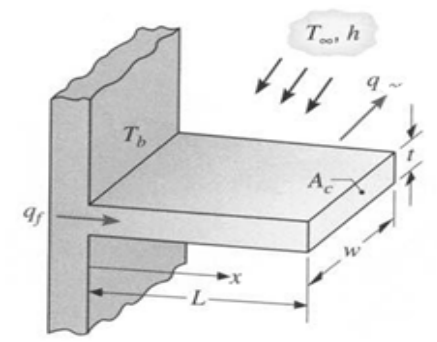

Fig. 1. Schematic diagram of longitudinal radiating fin 


\subsubsection{Development of the Governing Equation}

From the conservation of energy equation based on the assumptions

$$
\frac{d}{d x}\left(k(T) \frac{d T}{d x}\right)-\frac{2 \sigma \varepsilon}{\delta_{o}}\left(T^{4}-T_{\infty}^{4}\right)=0
$$

Since the thermal conductivity is assumed constant

$$
\frac{d^{2} T}{d x^{2}}-\frac{2 \sigma \varepsilon}{k \delta_{o}}\left(T^{4}-T_{\infty}^{4}\right)=0
$$

Which could also be written as,

$$
\frac{d^{2} T}{d x^{2}}-\frac{2 \sigma \varepsilon}{k \delta_{o}}\left(T-T_{\infty}\right)\left(T^{3}+T^{2} T_{\infty}+T T_{\infty}^{2}+T_{\infty}^{3}\right)=0
$$

The boundary conditions

$$
x=L, \quad-k \frac{\partial T}{\partial x}=h L\left(T-T_{\infty}\right), \quad x=0, \quad T=T_{b},
$$

In the reviewed literatures, numerical methods or approximate analytical methods were used in finding solutions to the kind of non-linear differential equation Eq. (6). Since, one of our major aims in this work is to generate a closed form solution for this equation, the following dimensionless parameters were used.

$$
\begin{aligned}
& B i=\frac{h L}{k} \quad X=\frac{x}{L} \quad \theta=\frac{T-T_{\infty}}{T_{b}-T_{\infty}}, \\
& B i_{m}=\frac{2 \sigma L^{2} \varepsilon}{k \delta}\left[\left(T^{3}+T_{\infty} T^{2}+T_{\infty}^{2} T+T_{\infty}^{3}\right]\right.
\end{aligned}
$$

On using the dimensionless parameters, the governing equations and boundary conditions transform to;

$$
\frac{d^{2} \theta}{d x^{2}}-B i_{m} \theta=0
$$

The boundary conditions are

$$
\begin{aligned}
& X=0, \theta=1 \\
& X=1, \frac{\partial \theta}{\partial X}=-B i \theta
\end{aligned}
$$

The Biot number as shown in the Eq. (9) gives a simple index of the ratio of the internal resistance of a body to conductive heat to its external resistance to convective heat. The modified Biot number in Eq. (10) gives a simple index of the ratio of the internal resistance of a body to conductive heat to its external resistance to radiative heat. This means that a small modified Biot number represent a small resistance to heat conduction, and thus small temperature gradients or rate of heat transfer within the fin. As part of our analysis, the use of different values of the Biot numbers (calculated using different values of thermal conductivity) represents different fin materials.

If negligible heat loss at the tip of the fin is considered, then boundary conditions are

$$
\begin{aligned}
& x=0, \quad T=T_{b}, \\
& x=L, \quad \frac{\partial T}{\partial x}=0
\end{aligned}
$$

Using the same dimensionless parameter as given in Eq. 8

$$
\begin{aligned}
& X=0, \quad \theta=1 \\
& X=1, \quad \frac{\partial \theta}{\partial X}=0
\end{aligned}
$$

On solving Eq. 9 with the boundary condition of Eq. 10

On solving equ. 11 with the boundary conditions of Eq. 12

$$
\begin{gathered}
\theta=\cosh \left(\left(\sqrt{B i_{m}} X\right)\right)-\left\{\left[\tanh \left(\left(\sqrt{B i_{m}}\right)(L)\right] \sinh \left(\left(\sqrt{B i_{m}}\right)(X)\right\}\right.\right. \\
\dot{Q}=\left(\sqrt{B i_{m}}\right) \sinh \left(\left(\sqrt{B i_{m}}\right)\right)-\left\{\left[\tanh \left(\left(\sqrt{B i_{m}}\right)(L)\right]\left(\sqrt{B i_{m}}\right) \cosh \left(\left(\sqrt{B i_{m}}\right)(X)\right\}\right.\right.
\end{gathered}
$$




$$
\eta=\left(1 /\left(\sqrt{B i_{m}}\right)\right) \sinh \left(\left(\sqrt{B i_{m}}\right)\right)-\left\{\operatorname { t a n h } \left(\left(\sqrt{B i_{m}}\right)(L) \mid\left(\left(1 / \sqrt{B i_{m}}\right)\right)\left(\cosh \left(\left(\sqrt{B i_{m}}\right)-1\right)\right\}\right.\right.
$$

Where, $\quad \dot{Q}=\frac{\dot{q} L}{K A\left(T_{b}-T_{a}\right)}$

\section{Results and Discussion}

Since a high thermal conductivity implies that energy can be conducted more easily, the results in the figures1-16 are as expected. The dimensionless temperature distribution falls monotonically along fin length for all various modified Biot number. For larger values of the modified Biot number $B i_{m}$, the more heat radiated from the fin through its length and the more thermal energy is

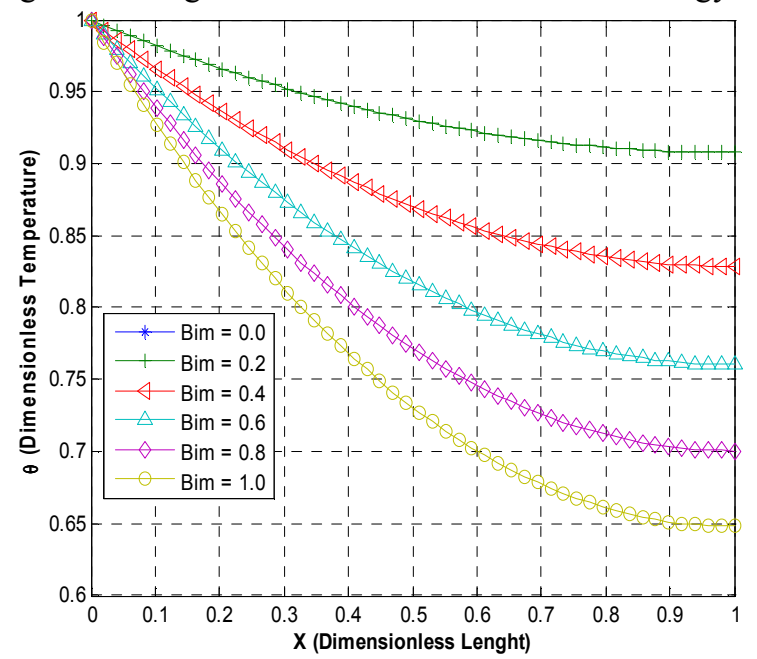

Fig. 2. Dimensionless Temp. Against Dimensionless length for $\mathrm{Bi}=0$

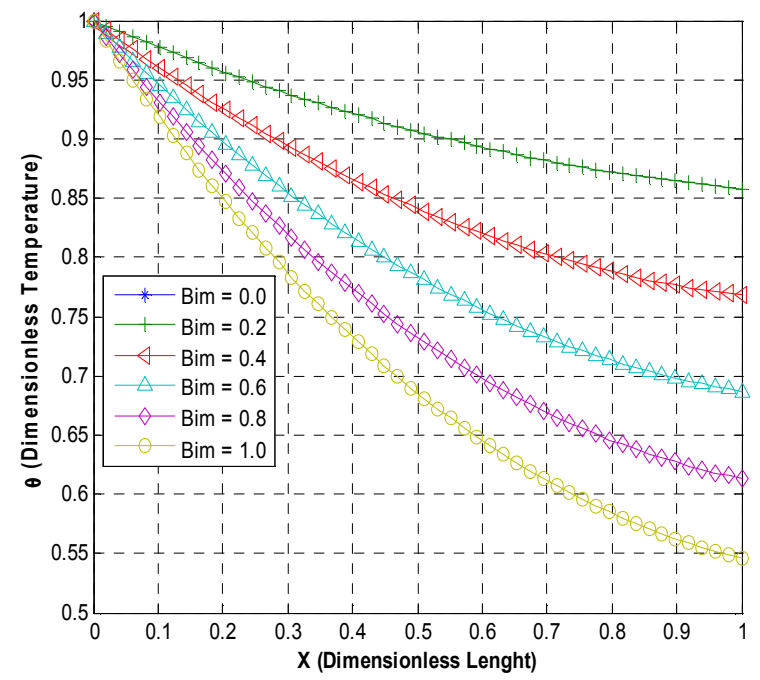

Fig. 4. Dimensionless Temp. Against Dimensionless length for $\mathrm{Bi}=0.04$ efficiently transferred into environment through the fin length. In the situation of negligible heat loss from the fin tip to the environment, the fin temperature decreases along the fin length also, and the temperature decreasing rate is the same around fin base area for all values of tip Biot numbers. This is due to heat which is majorly disposed to the surrounding through the fin tip. Also for high values of tip Biot number, the tip tends toward being isothermal and the thermal energy is efficiently transferred into the surrounding through the fluid-solid interface, hence, a lower temperature contour is expected as comparing to those with small values of tip Biot number.

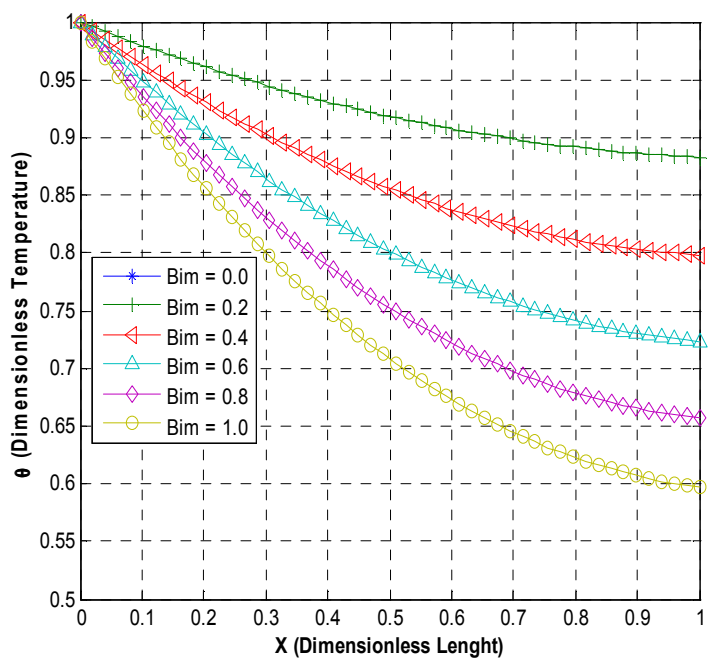

Fig. 3. Dimensionless Temp. Against Dimensionless length for $\mathrm{Bi}=0.02$

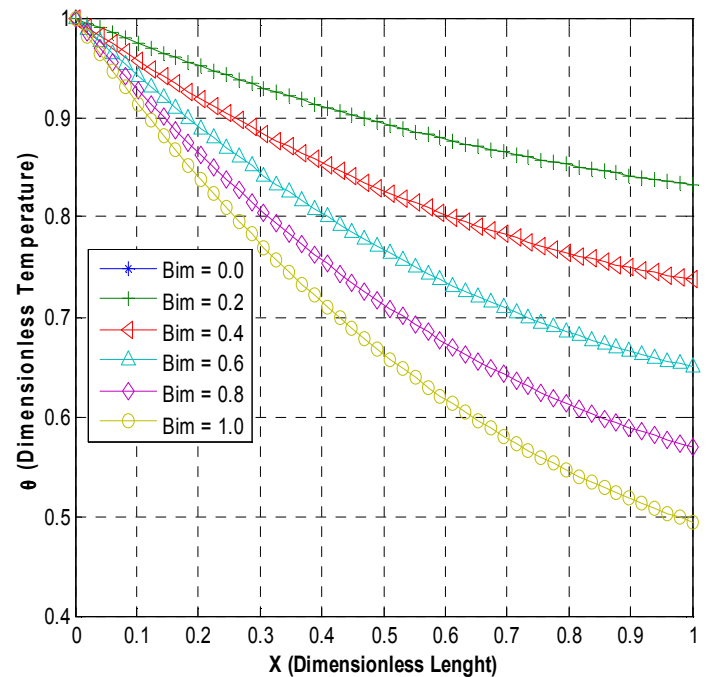

Fig. 5. Dimensionless Temp. Against Dimensionless length for $M=B i=0.06$ 


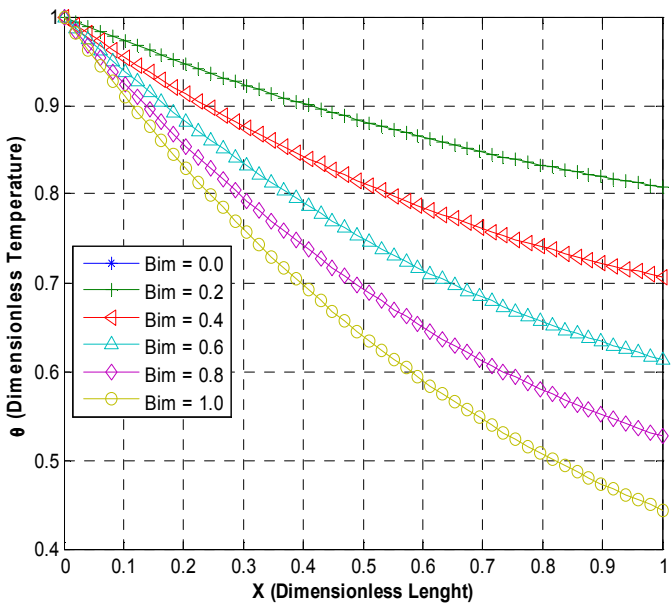

Fig. 6. Dimensionless Temp. Against Dimensionless length for $B i=0.08$

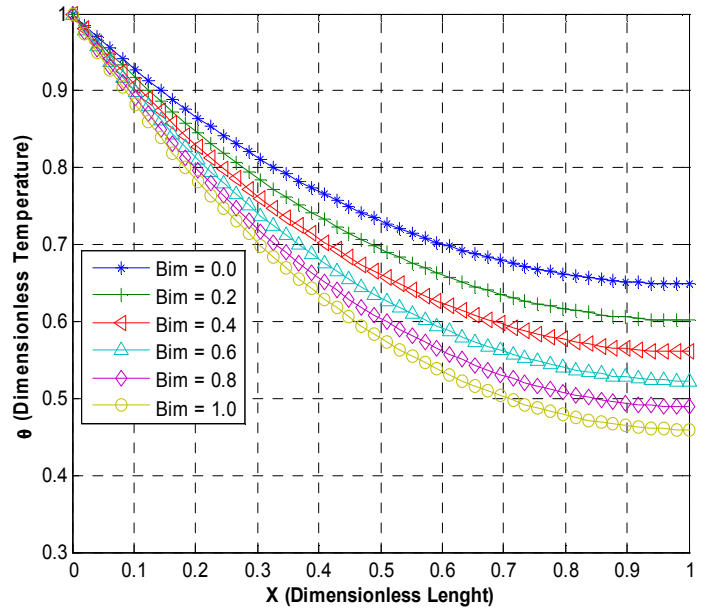

Fig. 8. Dimensionless Temp. Against Dimensionless length for $\mathrm{Bi}=0$

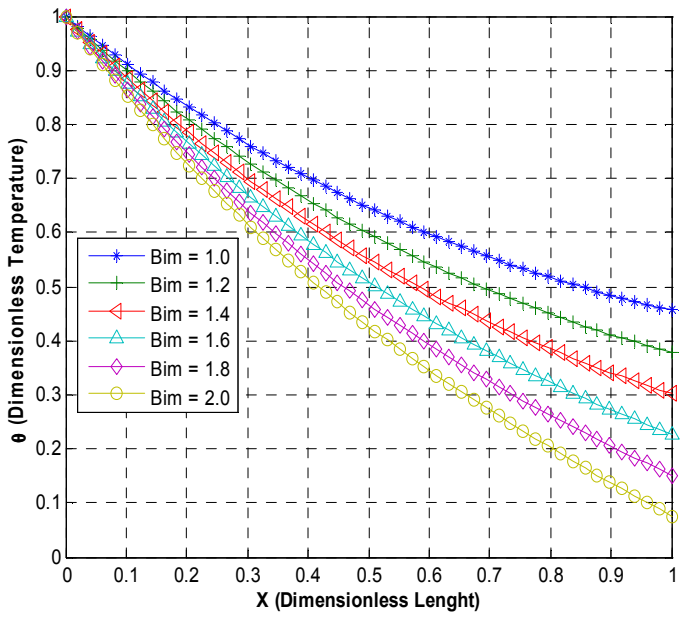

Fig. 10. Dimensionless Temp. Against Dimensionless length for Bi=0.075

Fig. 2 shows the variation of dimensionless temperature with dimensionless length and also the effect of the Modified Biot number on the straight fin with a negligible heat loss from the fin $\operatorname{tip}(B i=0)$. From the figure, as the Modified Biot number increases, the rate of heat transfer through the fin increases as the temperature in the fin drops

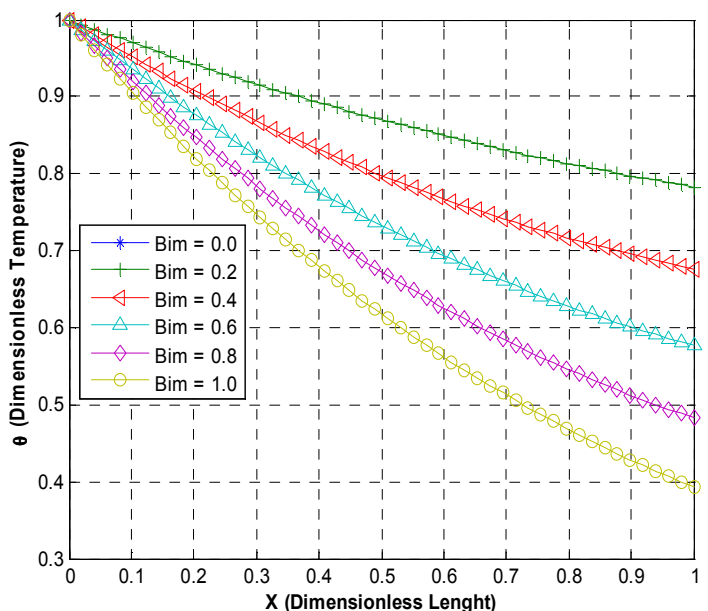

Fig. 7. Dimensionless Temp. Against Dimensionless length for $\mathrm{Bi}=0.1$

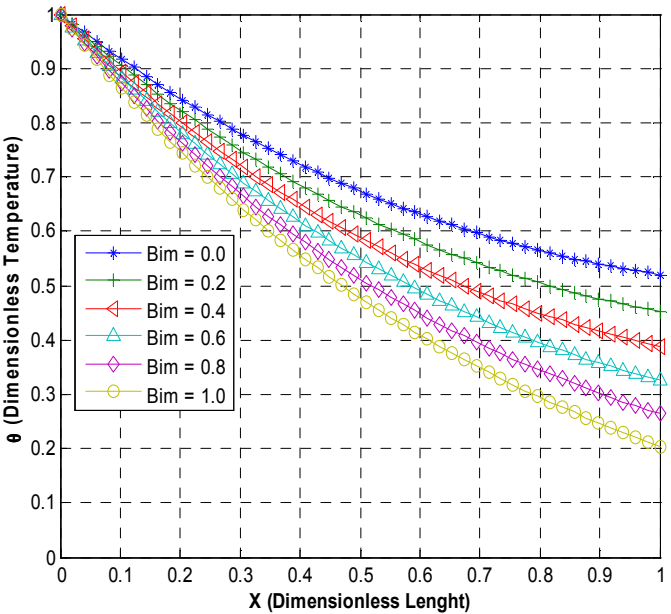

Fig. 9. Dimensionless Temp. Against Dimensionless length for $\mathrm{Bi}=0.05$

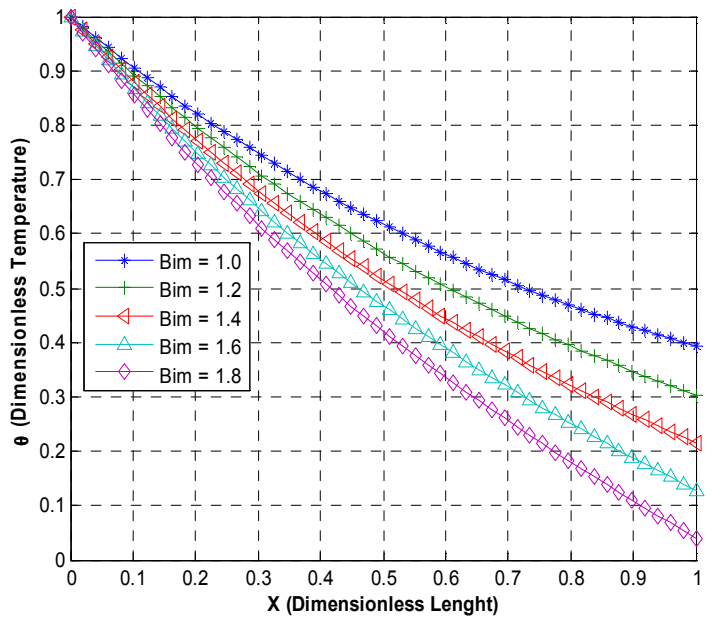

Fig. 11. Dimensionless Temp. Against Dimensionless length for $\mathrm{Bi}=0.1$

faster as depicted in the figure. The profile has steepest temperature gradient at $B i_{m}=1.0$, but its much higher value gotten from the lower value of thermal conductivity than the other values of $B i_{m}$ in the profiles produces a lower heat-transfer rate. This shows that the performance or efficiency of the fin is favoured at low values of Modified 
Biot number since our aim (high effective use of the fin) is to minimize the temperature decrease along the fin length, where the best possible scenario is when $\mathrm{T}=\mathrm{T}_{\mathrm{b}}$ everywhere.

Figures 3-11 show the variation of dimensionless temperature with dimensionless length in longitudinal radiating fin with convective tip. The effects of Biot number and Modified Biot number on the dimensionless temperature distribution and in consequent on the rate of heat transfer are shown. From the figures, it is shown that as the as the Biot number $(B i)$ and the Modified Biot number $\left(B i_{m}\right)$ increase, the rate of heat transfer through the fin increases. This is because as the fin radiative heat transfer increases and convective heat transfer increases at the tip of the fin, more heat is transferred by conduction through the fin thereby increases the temperature distribution in the fin and consequently the rate of heat transfer. Therefore, high effectiveness of fin could be achieved by using small values of Biot numbers, which could be realised using a fin of small length or by using a material of better thermal conductivity. Moreover, the results depict that care must be taken in the choice of length

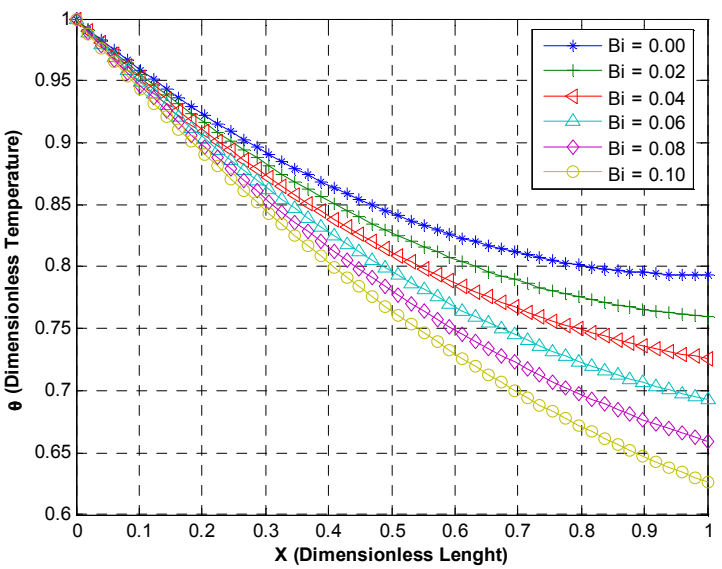

Fig. 12. Dimensionless Temp. Against Dimensionless for Bim=0.5

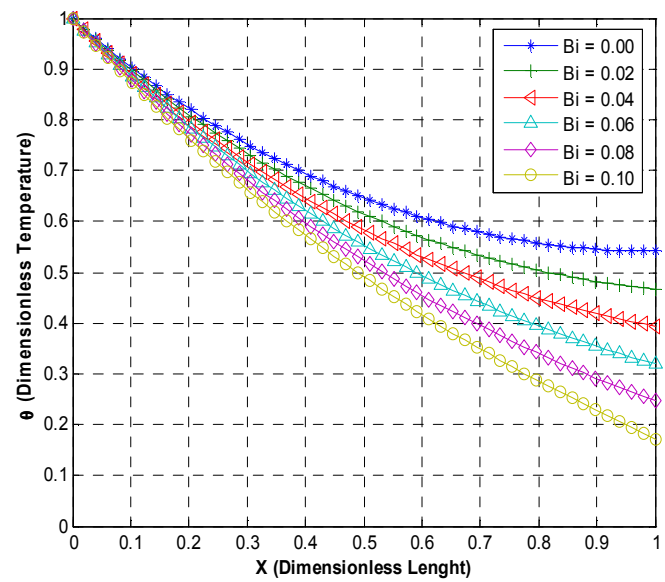

Fig. 14. Dimensionless Temp. Against Dimensionless length for Bim=1.5 of fin used during applications. This is because, as $B i_{m}$ (which increases as the fin length increases) tends to infinity, the fin efficiency tends to zero. The fin to a large extent of its length will remain at ambient! This consequently results in weak conduction limit. The extended area is largely useless in the heat transfer process and hence inefficient. Therefore, very long fins are to be avoided in practice. A compromise is reached for onedimensional analysis of fins $0<B i<0.1$. When the Biot number is greater than 0.1 , two dimensional analysis of the fin is recommended as one-dimensional analysis predicts unreliable results for such limit.

Figures 12-15 shows the effects of fin tip conditions on the dimensionless temperature distribution. From the figures, it could be seen that the at the value where the Modified Biot number equals 0.5 as shown in Fig.12, there was pronounced effects of fin tip condition on the temperature distribution along the fin length. As the value of the Modified Biot number increases, the effects of tip end condition vanishes as there are overlapping temperature distribution profiles as shown in fig. 13-15.

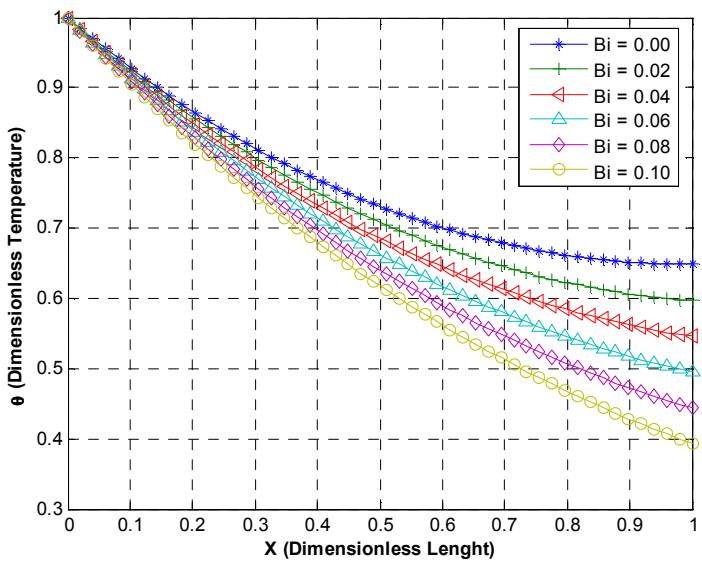

Fig. 13. Dimensionless Temp. Against Dimensionless for Bim=1.0

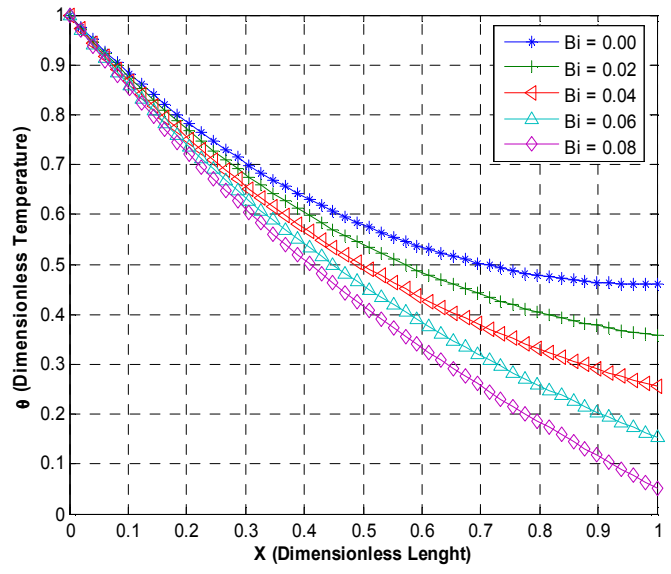

Fig. 15. Dimensionless Temp. Against Dimensionless length for Bim=2.0 


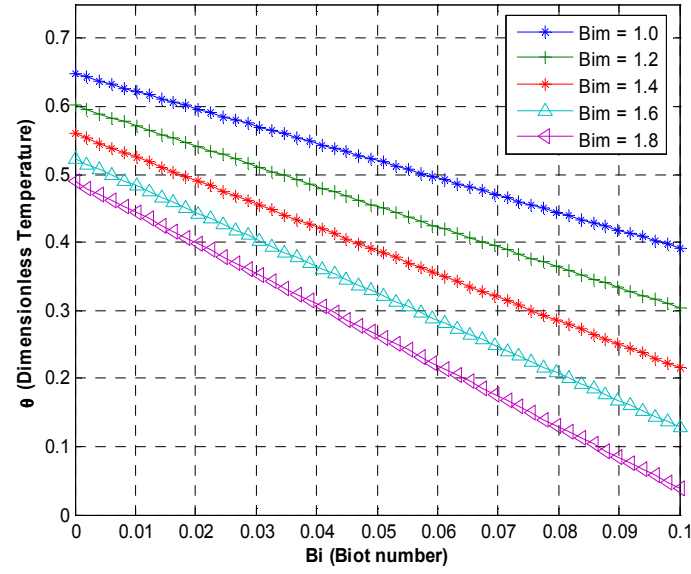

Fig. 16. Dimensionless Temp. Against Biot number

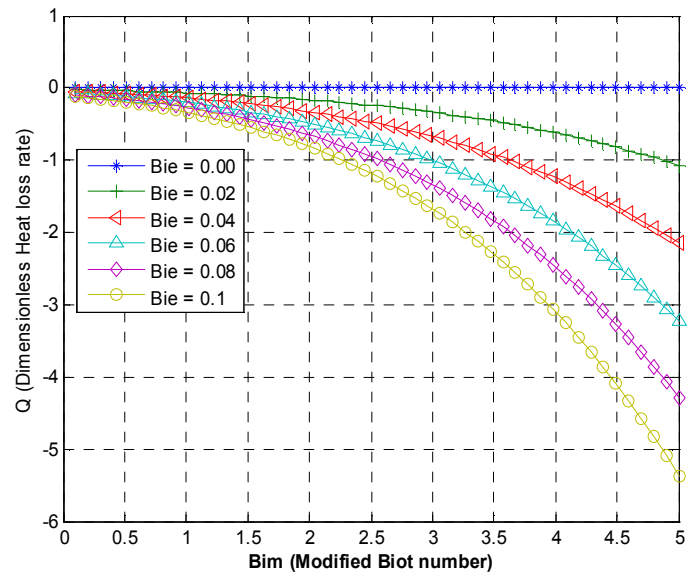

Fig. 18. Dimensionless Heat loss Against Modified Biot number

The effects of Biot number on the dimensionless temperature distribution for different Modified Biot number is shown in Fig. 16 while Fig. 17 shows the variation of dimensionless temperature distribution with Modified Biot number for different values of Biot number. In the limit $\mathrm{Bi} \rightarrow 0$, the fin heat transfer tends to zero even though the fin is entirely at the base temperature. This occurs when the heat transfer coefficient around the fin is relatively small as compared to the thermal conductivity of the fin and the fin length is also not too large. The effect of fin tip end condition on the rate of heat loss from the fin tip is shown Fig. 18. As it could be seen from the figure, as the Biot number increases, more heat is being convected away from the fin tip. Also, from Fig. 19 the fin efficiency decreases monotonically (for different Biot number) with increasing modified Biot number. The fin efficiency is unity in the limit $\mathrm{Bim} \rightarrow 0$. In this limit, the actual heat transfer rate from the fin is zero! This fin parameter plays a very important role in determining the amount of heat transfer from the fin as it accounts for the effects of decrease in temperature on the heat transfer from the fin. Since, the fin temperature drops along the fin length, the fin efficiency decreases with increase in fin length. Therefore, in practice required fin length should be properly determined because

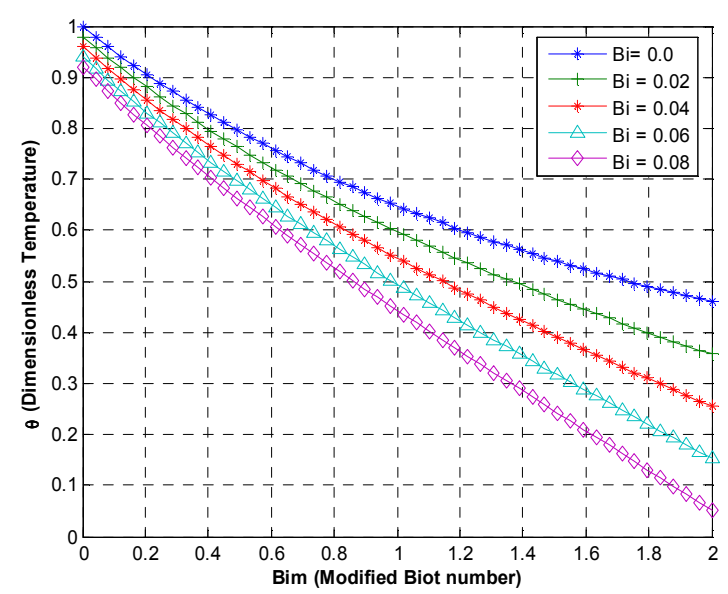

Fig. 17. Dimensionless Temp. Against Modified Biot number

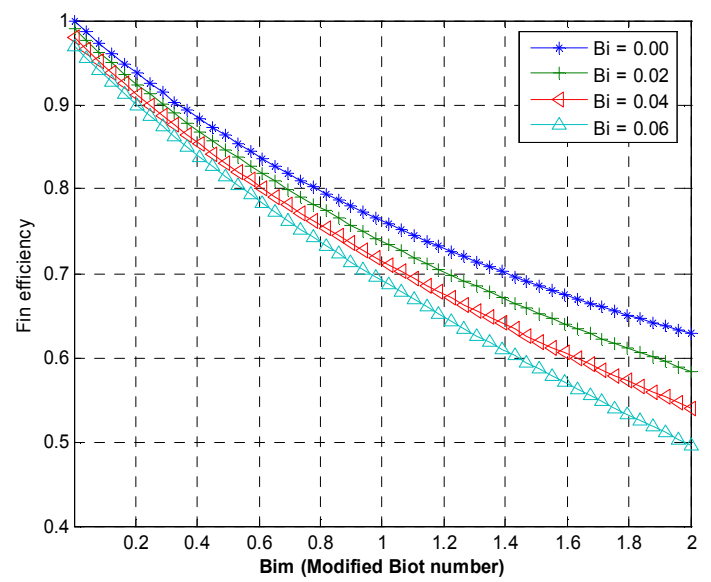

Fig. 19. Fin efficiency Against Modified Biot number

the fin length that causes the fin efficiency to drop below $60 \%$ usually cannot be justified economically and should be avoided.

\section{Conclusion}

In this work, temperature distribution in a straight fin having constant thermal conductivity with convecting and insulated tip has been solved analytically. The closed form solution was used to investigate the effects of the fin tip Biot number, Modified Biot number and tip end conditions on the temperature distribution, rate of heat transfer and efficiency of the straight. From the results, it is shown that the variation in the thermogeometric parameters (fin tip Biot number, Modified Biot number, and efficiency) of the fin has pronounced effects on the temperature distribution and consequently on the performance of the fin especially when a large temperature difference exists between the prime surface and the environment. Therefore, the total heat transfer, the fin effectiveness, and the fin efficiency are all enhanced when using a fin material with a high thermal conductivity. This depicts that the higher the thermal conductivity of the fin material, the better! For increase in the efficiency, the length of the fin could be decreased. 
This will decrease the total heat transfer area and the total energy transfer of a single fin, but multiple fins could be used to counter this effect, and still keep the efficiency high.

These results serve as basis for comparison of any other method of analysis of the problem and they also provide platform for improvement in the design of fin in heat transfer equipments such as air-land-space vehicles and their power sources, in chemical, refrigeration, and cryogenic processes, in electrical and electronic equipment, in conventional furnaces and gas turbines, in the design of firebox for the generation of steam power from fossil fuels.in process heat dissipators and waste heat boilers, and in nuclear-fuel modules, steam power plants, automobiles radiators etc.

\section{Nomenclature}

A cross sectional area of the fins, $\mathrm{m}^{2}$

Bi Biot number

$\mathrm{Bi}_{\mathrm{m}}$ Modified Biot number

$\mathrm{h}$ heat transfer coefficient, $\mathrm{Wm}^{-2} \mathrm{k}^{-1}$

$\mathrm{K}$ thermal conductivity of the fin $\mathrm{Wm}^{-1} \mathrm{k}^{-1}$

$\mathrm{L}$ Lenght of the fin, $\mathrm{m}$

M dimensionless fin parameter

$\mathrm{m}^{2}$ fin parameter $\mathrm{m}^{-1}$

$\mathrm{P}$ perimeter of the fin, $\mathrm{m}$

$\mathrm{T}$ Temperature, $\mathrm{K}$

$\mathrm{T}_{\mathrm{a}}$ ambient temperature, $\mathrm{K}$

$\mathrm{x}$ axial distance, $\mathrm{m}$

$\mathrm{X}$ dimensionless lenght

Q dimensionless heat transfer

$\eta \quad$ efficiency of the fin

\section{Greek Symbols}

$\delta \quad$ thickness of the fin, $m$

$\varepsilon$ emissivity of the fin

$\theta$ dimensionless temperature

$\sigma \quad$ Stefan-Boltzmann constant

$\delta_{b} \quad$ fin thickness at its base.

\section{References}

[1] R.D. Cockfield, Structural optimization of a space radiator, J. Spacecraft Rockets 5 (10) (1968) 1240-1241.

[2] J.E. Jr Wilkins Minimizing the Mass of Thin Radiating Fins, J. Aerospace Science, Vol. 27, 1960, 145-146.

[3] N. M. Schnurr, "Radiation from an array of longitudinal fins of triangular profile", AIAA Journal, Vol. 13, 1975, 691-693.

[4] S. P. Venkateshan; Gopinath, Ashok, "Asymptotic analysis of a uniform area radiating fin", Canadian Society for Mechanical Engineering, Transactions, Vol. 11, No. 2, 1978,103-108.

[5] T. J. Love and J. E. Francis, A linearized analysis for longitudinal fins with radioactive and convective exchange Thermo physics and Heat Transfer Conference, Palo Alto, Calif., AIAA, 1978.

[6] R. D. Karam and R. J. Eby, "Linearized solution of conducting radiating fins," AIAA Journal (Fluid Mechanics and Heat Transfer), Vol. 16, 1978, 536-538.

[7] N. M. Schnurr.; M. A. Townsend.; A. B. Shapiro, "Optimization of radiating fin arrays with respect to weight", ASME, Transactions, Series C - Journal of Heat Transfer, Vol. 98, 1976, 643-648.

[8] R. G. Eslinger and B. T. F. Chung. Periodic heat transfer in radiating and convecting fins or fin arrays, American Institute of Aeronautics and Astronautics and American Society of Mechanical Engineers, Thermo physics and Heat Transfer Conference, Palo Alto, Calif., AIAA ,1978.

[9] H. V. Truong and R. J. Mancuso, Performance predictions of radiating annular fins of various profile shapes, American Society of Mechanical Engineers and American Institute of Chemical Engineers, Joint National Heat Transfer Conference, Orlando, Fla., ASME 5, 1980.

[10] H. V. Chang, Optimization of a heat pipe radiator design, Thermo physics Conference, Snowmass, CO, 1984.

[11] F. G. Zaulichnyi, Possibilities for improving the characteristics of a radiator cooler through the use of finned heat pipes as radiating elements, Sibirskii FizikoTekhnicheskii Zhurnal (ISSN 0869-1339), Category: Fluid Mechanics and heat transfer, 1991,20-26.

[12] S. Sunil Kumar and Venkateshan S. P. "Optimized Tubular Radiator with Annular Fins on a Non-Isothermal Base, Int. J. Heat and Fluid Flow", Vol.15, 399-409, 1994.

[13] R. N. Krikkis and R. Panagiotis Optimum Design of Spacecraft Radiators with Longitudinal Rectangular and Triangular Fins, J. Heat Transfer, Vol.124, 805-811, 2002.

[14] R. J. Naumann, Optimizing the design of space radiators, Int J. Thermophys. 25 (2004)1929-1941

[15] A. Cihat, Optimum design of space radiators with temperature-dependent thermal conductivity, Applied Thermal Engineering 26 (2006) 1149-1157.

[16] M. J. Hosseini, M. Gorji and M. Ghanbarpour, Solution of Temperature Distribution in a Radiating Fin Using Homotopy Perturbation Method, Mathematical Problems in Engineering Volume 2009, Article ID 831362.

[17] F. Bazdidi-Tehrani, and M. H. Kamrava, Combined Heat Transfer Calculations in a Fin-Tube Radiators with Temperature-dependent Thermal Conductivity, International Conference on Mechanical and Industrial Engineering, WASET, (2010), Singapore, pp176-184. 